University of Nebraska - Lincoln

DigitalCommons@University of Nebraska - Lincoln

Center for Brain, Biology and Behavior: Papers \&

Publications

Brain, Biology and Behavior, Center for

2019

Dynamic effects of habituation and novelty detection on newborn event related potentials

Cathryn S. Cortesa

Caitlin M Hudac

Dennis Molfese

Follow this and additional works at: https://digitalcommons.unl.edu/cbbbpapers

Part of the Behavior and Behavior Mechanisms Commons, Nervous System Commons, Other Analytical, Diagnostic and Therapeutic Techniques and Equipment Commons, Other Neuroscience and Neurobiology Commons, Other Psychiatry and Psychology Commons, Rehabilitation and Therapy Commons, and the Sports Sciences Commons

This Article is brought to you for free and open access by the Brain, Biology and Behavior, Center for at DigitalCommons@University of Nebraska - Lincoln. It has been accepted for inclusion in Center for Brain, Biology and Behavior: Papers \& Publications by an authorized administrator of DigitalCommons@University of Nebraska Lincoln. 


\title{
Dynamic effects of habituation and novelty detection on newborn event related potentials
}

\author{
Cathryn S. Cortesa, ${ }^{1,2}$ Caitlin M. Hudac,, \\ \& Dennis L. Molfese ${ }^{1,2}$ \\ 1 Department of Psychology, University of Nebraska-Lincoln, 238 Burnett Hall, \\ Lincoln NE 68588, USA \\ 2 University of Nebraska-Lincoln Center for Brain, Biology and Behavior, \\ C89 East Stadium, Lincoln NE 68588, USA \\ Corresponding author - C.S. Cortesa, Johns Hopkins University, Department of \\ Cognitive Science, 237 Krieger Hall, 3400 N. Charles St, Baltimore, MD 21218, USA; \\ email cathryn.cortesa@gmail.com
}

\begin{abstract}
Newborns habituate to repeated auditory stimuli, and discriminate syllables, generating opportunities for early language learning. This study investigated trial-bytrial changes in newborn electrophysiological responses to auditory speech syllables as an index of habituation and novelty detection. Auditory event-related potentials (ERPs) were recorded from 16 term newborn infants, aged 1-3 days, in response to monosyllabic speech syllables presented during habituation and novelty detection tasks. Multilevel models demonstrated that newborns habituated to repeated auditory syllables, as ERP amplitude attenuated for a late-latency component over successive trials. Subsequently, during the novelty detection task, earlyand late-latency component amplitudes decreased over successive trials for novel syllables only, indicating encoding of the novel speech syllable. We conclude that newborns
\end{abstract}

Published in Brain and Language 199 (2019) 104695

doi:10.1016/j.bandl.2019.104695

Copyright (C) 2019 Elsevier Inc. Used by permission.

Submitted 4 October 2018; revised 28 August 2019; accepted 5 September 2019. 
dynamically encoded novel syllables over relatively short time periods, as indicated by a systematic change in response patterns with increased exposure. These implications for understanding early precursors of learning and memory in newborns.

Keywords: Habituation, Newborn, Event-related potentials, Novelty detection, Auditory

\section{Introduction}

The world is an inherently complex environment in which infants are continually being exposed to repeating and increasingly familiar elements (e.g. language sounds or a caregiver's face) and new objects, agents, sounds, and textures. For over 50 years, the study of neonatal habituation to a repeated stimulus (e.g. Bridger, 1961; Fantz, 1964; Swain, Zelazo, \& Clifton, 1993) has been used to describe neuropsychological and cognitive functioning in early infancy. Classic behavioral studies use simple infant behaviors such as looking time, sucking rates, and head turning as indices of infant habituation (Oakes, 2010). However, there are challenges to the interpretation of such approaches, including individual differences in the amount of exposure required to reach habituation criterion (i.e. Colombo, Mitchell, Coldren, \& Freeseman, 1991), competing familiarity and novelty preferences (i.e. Hunter \& Ames, 1988), interfering variables such as stimulus complexity, dynamics, and a priori stimulus preferences unrelated to habituation (i.e. Quinn, Yahr, Kuhn, Slater, \& Pascalis, 2002), and observer inference as well as the general uncertainty of using external evidence to infer about an infant's mental state or stimulusdetection threshold (i.e. Nizami, 2019a, 2019b). Initially, under most conditions, infants look longer at a familiar stimulus than a novel one. After a certain period of time, which varies in length based on many factors including infant age and stimulus complexity, visual preference undergoes a familiarity-novelty shift, whereby the infant transitions to longer looking times to the novel stimulus (Rose, Gottfried, MelloyCarminar, \& Bridger, 1982). However, when no a priori preference is expected, researchers may induce familiarity of one stimulus through pre-experimental exposure, or habituation. In this case, during the post habituation testing phase, infants typically demonstrate an initial preference for novelty (Houston-Price \& Nakai, 2004). Infant novelty and familiarity preferences have complex discontinuities 
and non-linearities, as part of a dynamical system of infant cognition, such that changes in experimental paradigm or stimulus parameters can drastically alter infants' preference for familiar or novel stimuli (Schöner \& Thelen, 2006).

The mechanisms by which habituation and novelty detection occur in humans are addressed primarily by two theories: the stimulus-comparator theory, and the dual process theory. According to the stimulus comparator theory (Kavšek, 2013; Sokolov, 1963b), attention is first directed to a stimulus using the orienting reflex (Colombo \& Mitchell, 2009). The orienting reflex physiologically prepares the infant to take in information (e.g., listening to maternal voice) by directing sensory mechanisms and receptors toward the stimulus and reducing other physiological activity, including suppressing heart rate and motor activity (Sokolov, 1963a; Sokolov, 1966). Subsequent incoming stimuli are compared against the existing encoded mental representation (Colombo \& Mitchell, 2009). Novel stimuli elicit greater orienting reflexes relative to familiar stimuli, inversely proportional to the strength of the encoded mental representation (Colombo \& Mitchell, 2009). Although the dual process theory of habituation predicts a similar decrease in responding as a result of repeated exposures, it is thought to be driven by simple mechanisms of excitation and inhibition that occur along two distinct pathways (Groves \& Thompson, 1970). First, stimulus-specific neural processing decreases the responsiveness over repeated exposures, resulting in habituation and learning. Second, a nonspecific sensitization process produces arousal that corresponds to the stimulus strength or salience. The dual process theory suggests that highly activating or salient stimuli will create an initial increase in responding as a result of the sensitization mechanism. This initial increase will be followed by a decrease in responding, as the habituation mechanism grows sufficiently strong to overpower the sensitization mechanism (Groves \& Thompson, 1970).

To better understand the underlying neural mechanisms involved in habituation and novelty detection, investigators have turned to brain imaging to investigate auditory habituation (e.g. Rosburg \& Sörös, 2016). One example is the event-related potential (ERP), a portion of the ongoing electroencephalograph (EEG) that is time-locked to the onset of a stimulus event. ERPs are effective to study changes in neural activity during habituation tasks across development, from adults 
(Megela \& Teyler, 1979; Wastell \& Kleinman, 1980a, b) to children (Hudac et al., 2018; Regtvoort, van Leeuwen, Stoel, \& van der Leij, 2006), and infants (i.e. Gonzalez-Frankenberger et al., 2008; Nikkel \& Karrer, 1994). ERPs are optimal for studying newborns. They are safe, painless, and do not require a behavioral response (Johnson et al., 2001; Picton \& Taylor, 2007; Wolfe \& Bell, 2007), although newborn ERP waveform morphology does not contain traditional adultlike peak components such as the $\mathrm{P}_{3}$ or $\mathrm{N} 2$ and changes drastically in the first year of life (Kushnerenko, Čeponiene, Balan, Fellman, \& Näätänen, 2002). ERPs also provide temporally precise information on a millisecond time scale that can measure the dynamic trialby-trial changes in neural processing mechanisms and associated behaviors over time (Csibra, Kushnerenko, \& Grossmann, 2008).

Previous research demonstrates that neonatal ERPs differentiate novel from familiar stimuli (Ruusuvirta, Huotilainen, Fellman, \& Näätänen, 2004; Snyder, Garza, Zolot, \& Kresse, 2010). In addition, ERPs recorded from newborns may predict later language and cognitive developmental outcomes (Fellman et al., 2004; Molfese, Molfese, \& Modgline, 2001; Tsao, Liu, \& Kuhl, 2004), as well as the development of learning disabilities including dyslexia and other cognitive abilities such as reading ability (Guttorm, Leppänen, Hamalainen, Eklund, \& Lyytinen, 2010; Leppänen et al., 2012; Molfese, 2000). ERPs may hold the key to better understanding the dynamic cognitive mechanisms underlying newborn habituation. However, much is still unknown about the mechanisms of typical neonatal cognition. Therefore, interpretation of indicators of risk for later developmental disabilities is difficult, and more basic research is needed to explicate neonatal cognitive mechanisms of habituation and novelty detection.

Few studies utilize neuroimaging to jointly examine both habituation and novelty detection at the earliest developmental stages, with notable exceptions examining neonates within the first few days after birth (Mahmoudzadeha et al., 2013; Matuz et al., 2012; Muenssinger et al., 2013). In addition, researchers have used ERPs to assess novelty detection in newborns (Čeponiene et al., 2002) and infants (Kushnerenko, Van den Bergh, \& Winkler, 2013; Snyder et al., 2010; Thomas, Shucard, Shucard, \& Campos, 1989), as well as to discriminate deviant stimuli from a standard pattern or the mismatch negativity at birth (MMN) (Carral et al., 2005; Ruusuvirta et 
al., 2004). Similar to behavioral studies of novelty detection, neonates typically demonstrate an increase in neural response amplitude in response to novel stimuli, or a change from a standard pattern, consistent with the requisite characteristics of habituation (Rankin et al., 2009; Thompson \& Spencer, 1966).

In typical habituation paradigms, a gradual within-session change in response serves as the experimental outcome with the expectation that subjects' response will change from the beginning to the end of a habituation paradigm. The shift from initial familiarity preference to novelty preference is thought to occur, at least partially, as a result of accumulated experience with the stimuli (Hunter, Ames, \& Koopman, 1983; Roder, Bushnell, \& Sasseville, 2000). However, ERP signals are traditionally averaged across all the experimental trials to create a mathematical average response in order to improve signalto-noise ratios (Little, Thomas, \& Letterman, 1999). This is an inherent contradiction for evaluating within-session changes in response to repeated stimuli. To address this problem, other researchers have implemented "fast habituation" research designs (e.g., Wastell \& Kleinman, 1980a, b) or tested split-half comparisons. For instance, between 6 and 9 months, infants exhibit neural adaption as reflected by a reduction in ERP component amplitude during early (averaged) trials compared to later (averaged) trials (Nikkel \& Karrer, 1994; Snyder, Webb, \& Nelson, 2002; Wiebe et al., 2006). However, split-half averaging cannot address spontaneous, dynamic mechanisms underlying the responses (Blankertz, Lemm, Treder, Haufe, \& Muller, 2011; Turk-Browne, Scholl, \& Chun, 2008). Recent work has utilized single-trial analysis of ERPs in adults (e.g. Amsel, 2011; Tremblay \& Newman, 2015; Wichary, Magnuski, Oleksy, \& Brzezicka, 2014), adolescents (e.g. Bender et al., 2015; Milne, 2011), and infants (e.g. Hofmann, Salapatek, \& Kuskowski, 1981; Little et al., 1999).

Thus, this study aimed to use a data-driven approach to first identify temporal regions of variability in neonatal ERPS, and then to incorporate the micro time scale of single-trials by accounting for the sequential position of each trial. We opted to evaluate the effect of within-session change using trial-by-trial analysis via multilevel models (Hoffman, 2007; Hoffman \& Stawski, 2009) that track the ongoing linear or nonlinear changes in ERP responses without relying on blockstyle averaging. We assume background noise to be random, and 
we test whether measuring a large number of individual trials per participant, as opposed to a small number of average ERPs, will allow statistical models sufficient power to detect trial-by-trial changes in the underlying brain activity.

Our study targeted habituation and novelty detection of speech sounds, which are essential for the development of speech and language (Streri, Hevia, Izard, \& Coubart, 2013). Although less is known about neural networks for language at birth compared to later in development, we predicted bilateral speech discrimination (i.e., discrimination across both left and right hemispheres) consistent with other work in neonates (Molfese, 2000) and older developmental populations (Perani et al., 2011).

We presented monosyllabic speech syllables to newborn infants as part of a habituation paradigm. First, a single syllable was played repeatedly to assess habituation, and subsequently this now familiar syllable was randomized with an equal number of a novel syllable in order to test for novelty detection. Using a systematic analytic approach, we evaluated the specific ERP patterns that index habituation (Experiment 1) and novelty detection (Experiment 2). We hypothesized different patterns of amplitude change for both experiments: In Experiment 1, we predicted that the response amplitude to a single, repeated stimulus would decrease across successive trials. In Experiment 2, we predicted that the ERP amplitude to the familiar stimulus (i.e., speech sound from Experiment 1) would remain stable (i.e., not change over successive trials), while ERP amplitude to a novel speech sound would decrease across successive trials. Finally, based on previous research investigating hemisphere laterality differences to speech sounds in newborns (Molfese \& Molfese, 1979), we hypothesized different polarity of ERP response (positive or negative) between left and right hemisphere electrode regions, but no absolute amplitude differences between hemispheres. To address these hypotheses, we conducted a two-stage analysis for each experiment. First, we identified temporal windows of interest within the ERP waveform using a data driven principal component analysis to assess variability, considering that newborn ERP morphology does not consist of traditional ERP "peaks". Second, we assessed the sequential changes in amplitude across successive trials using linear models to examine trialby-trial change in newborn's ERP responses. 


\section{Material and methods}

All procedures were approved by both the local university and the hospital ethical committees.

\subsection{Participants}

Participants and their parents were recruited from the hospital nursery in a Midwestern state in the United States. Twenty-six infants participated, but six were excluded because of insufficient artifact free trials (i.e. for at least one of the experimental conditions, $<1 / 3$ of the trials were free from artifacts), three due to interruptions during the testing session, and one due to counterbalancing error. The final sample consisted of sixteen healthy full-term newborns ( $8 \mathrm{fe}$ male) between 36and 42-weeks gestational age. Fourteen $(87.50 \%)$ were vaginal births, while two (12.50\%) were cesarean births. Two (12.50\%) identified as Hispanic or Latino, and fourteen $(87.50 \%)$ identified as white non-Hispanic. All participants passed hospital administered otoacoustic emissions newborn hearing screening in both ears and were born to English-speaking households. At the time of testing, the infants were less than three days old. Parents provided informed consent. Additional demographic information is reported in Table 1.

\subsection{Auditory stimuli}

The computer-synthesized monosyllabic consonant-vowel stimuli employed were generously provided by Drs. Stevens and Blumstein (Stevens \& Blumstein, 1978). The stimuli have been described

Table 1 Sample demographic characteristics $(\mathrm{N}=16)$.

\begin{tabular}{lrr} 
& Mean & $S D$ \\
\hline Gestational Age (weeks) & 39.16 & 0.94 \\
Birth Weight (kg) & 3.53 & 0.35 \\
APGAR (1 min) & 7.85 & 0.72 \\
APGAR (5 min) & 8.69 & 0.70 \\
APGAR (10 min) & 8.81 & 0.40 \\
Maternal Age & 28.88 & 5.15 \\
Paternal Age & 29.31 & 5.28 \\
\hline
\end{tabular}


in previous studies (see Key et al., 2007). For the present study, one of two speech stimulus sets was used (stimulus set 1:/da/and /ga/; stimulus set $2: / \mathrm{du} /$ and $/ \mathrm{gu} /$ ). Stimulus set 1 starting frequencies of F2 and F3 were $1580 \mathrm{~Hz}$ and $2680 \mathrm{~Hz}$, respectively, (token 7). Stimulus set 1 starting frequencies of $\mathrm{F}_{2}$ and $\mathrm{F} 3$ were $1600 \mathrm{~Hz}$ and 2700 $\mathrm{Hz}$, respectively, (token 13). Each syllable was edited to $300 \mathrm{~ms}$ duration and matched in peak loudness level.

\subsection{Auditory habituation task}

Speech syllables were presented at $80 \mathrm{~dB}$ SPL(A) measured at the infant's ear. Stimuli were presented using E-prime version 2 (Psychology Software Tools, Pittsburgh, PA). All stimuli were presented via an overhead 8 Ohm speaker positioned $1 \mathrm{~m}$ over the midline of the newborn's head while swaddled in their hospital bassinet. In Experiment 1, 40 consecutive repetitions of one speech syllable (e.g.,/da/) were presented in order to induce habituation. Following a quiet 10-second delay, Experiment 2 was then presented with equiprobable randomized presentations of either the familiar speech syllable (e.g.,/da/) or the novel, paired speech syllable (e.g., /ga/) for 40 trials each for a total of 80 trials. Stimuli were presented with randomly varying inter-stimulus intervals between 1100 and $1300 \mathrm{~ms}$.

\subsection{Electrophysiological recording}

ERP data was collected in a darkened, quiet room in the hospital nursery. Newborns were comfortably swaddled, and placed in a basinet propped at an angle of approximately $30^{\circ}$ such that their head was inclined in order to help maintain a quiet alert state of consciousness during testing. Researchers continuously monitored the ongoing EEG activity to ensure that stimulus presentation occurred when the newborn was in a quiet alert state during data collection. Stimulus presentation was paused during periods of motor movements (at least two seconds of visible movement artifacts in the EEG data) and where the EEG indicated that the infant was sleeping as indexed by large slow wave EEG activity. Stimulus presentation was resumed when the newborn's behavior and ongoing EEG indicated a quiet alert state (at least two seconds of visibly artifact-free EEG data). 
EEG was recorded using a 128-channel AgAgCl electrode Hydrocel Geodesic Sensor Net and NetStation 4.4.2 software sampled at $250 \mathrm{~Hz}$ using high-impedance amplifiers (Philips Neuro, Eugene, $\mathrm{OR}) . \mathrm{Cz}$ (vertex) reference was used during recording. Electrode impedances recorded before and after the task were below $60 \mathrm{KOhms}$ to maximize signal-to-noise ratio. After data collection, the ERP data were filtered, segmented, and cleaned using the NetStation Waveform tools. Unsegmented data were first filtered using a bandpass of 0.3$30 \mathrm{~Hz}$. Next, trials were segmented from the continuous EEG data to include a $100 \mathrm{~ms}$ baseline period and extended $700 \mathrm{~ms}$ post-stimulus, adjusting for computer timing offsets (measured monthly) and digital finite impulse response (FIR) filters. In the case of voltage shifts greater than $150 \mu \mathrm{V}$ (e.g. motor artifacts), signals were classified as artifacts and corrected using spline interpolation from immediately adjacent electrodes using Net Station Waveform tools algorithms (Ferree, 2000). On average, 25\% of trials were excluded due to artifacts, and the remaining trials were included in the analysis. Each newborn contributed an average of 30 trials per each of the three conditions to the analysis (Habituation $M=30.19$, $S D=6.61$ (range: 14-37), Familiar: $M=29.75, S D=6.10$ (range: $17-38$ ), Novel: $M=$ 29.94, $S D=5.65$ (range: $17-39$ )). ERPs were baseline corrected using the 100 ms baseline before stimulus onset, re-referenced to the average of all electrodes, and averaged separately for each experiment and of the two stimulus conditions again using NetStation waveform tools. For analyses, the 128-electrode array was clustered into five bilateral scalp electrode clusters (5: frontal, central, temporal, parietal, and occipital) across each hemisphere to create 10 electrode clusters (Molfese, Tan, Sarkari, \& Gill, 1997), exact details of which can be found in supplemental materials. This step increased statistical power by reducing electrode locations to a number of homologous scalp clusters (Curran, 1999).

\subsection{Analytic strategy}

Our approach combines the classic use of temporal principal components analysis (tPCA) to identify temporal windows of interest within the ERP (Hudac, Cortesa, Ledwidge, \& Molfese, 2018; Molfese, Nunez, Seibert, \& Ramanaiah, 1976) with modern multilevel modeling 
techniques to characterize patterns of change across the experiment, similar to work conducted in special pediatric populations (e. g. Hudac et al., 2018). Analyses for Experiments 1 and 2 followed similar procedures.

2.5.1. Temporal window selection via temporal principal components analysis (tPCA)

First, temporal components of the group average ERP waveforms were identified using a temporal principal component analysis (tPCA) as a variable-reduction technique in SPSS version 23.0 (IBM, Chicago, IL). This approach is based on a body of previous research which demonstrates that analysis of latent components of the ERP waveforms can optimize data-driven representation of electrophysiological data (Donchin, 1966; Kayser \& Tenke, 2003), especially for infants (i.e. Molfese, Nunez, Seibert, \& Ramanaiah, 1976). We analyzed the data separately for each experiment, but followed the same analytic strategy across both experiments, outlined here. Because the data was recorded at a rate of $250 \mathrm{hz}, 175$ time point variables were recorded, one every four ms from o to $700 \mathrm{~ms}$ post-stimulus onset. The tPCA analysis used the FACTOR procedure with each of the 175 timepoints as variables, using varimax rotation and a correlation matrix. The data structure for the TPCA analysis contained averaged ERP amplitude values for each of the 175 time points, for each participant, for each of the 10 scalp clusters, and in Experiment 2 for each stimulus condition. First, the tPCA was conducted to extract 15 temporal components from the 175 original time points, and the Scree test (Cattell, 1966) was used to determine the number of components to retain. Then, we ran the IPCA a second time using the number of components retained by the Scree test.

\subsubsection{Characterization of temporal windows}

For each retained component, temporal windows were identified as portions of the waveform accounting for the greatest variance. Specifically, the duration of the time window for the temporal component is defined as the set of contiguous time points at which the principal component score is greater than 0.6o. Each temporal component (TC) is reported in order from stimulus onset (i.e., temporal order, not factor order) and named based upon experiment $(\mathrm{H}=$ 
Table 2 Temporal components (TC) characterization.

\begin{tabular}{|c|c|c|c|c|c|}
\hline & \multirow{2}{*}{$\begin{array}{l}\text { \%Total } \\
\text { Variance }\end{array}$} & \multicolumn{2}{|c|}{ Variance Latency } & \multicolumn{2}{|l|}{ Topography characterization by voltage } \\
\hline & & Range & Peak & Positive & Negative \\
\hline \multicolumn{6}{|c|}{ Experiment 1: Habituation (H) TCs } \\
\hline Overall & $94 \cdot 79 \%$ & & & & \\
\hline HTC1 & $16.20 \%$ & $4^{-120 \mathrm{~ms}}$ & $36 \mathrm{~ms}$ & Parietal & Frontal, Central \\
\hline HTC2 & $16.74 \%$ & $112-252 \mathrm{~ms}$ & $188 \mathrm{~ms}$ & & Central, Temporal \\
\hline $\mathrm{HTC}_{3}$ & $24.40 \%$ & $244-460 \mathrm{~ms}$ & $344 \mathrm{~ms}$ & Frontal, Central & Temporal, Occipital \\
\hline $\mathrm{HTC}_{4}$ & $37 \cdot 45 \%$ & $404-700 \mathrm{~ms}$ & $636 \mathrm{~ms}$ & Parietal, Occipital & Frontal, Central, Temporal \\
\hline \multicolumn{6}{|c|}{ Experiment 2: Novelty detection (N) TCs } \\
\hline Overall & $96.74 \%$ & & & & \\
\hline NTC1 $1 \S$ & $1.91 \%$ & & & & \\
\hline NTC2 & $30.23 \%$ & $4-232 \mathrm{~ms}$ & $72 \mathrm{~ms}$ & Right Central, Right Parietal & Left Frontal, Left Temporal \\
\hline $\mathrm{NTC}_{3}$ & $27.19 \%$ & $224-476 \mathrm{~ms}$ & $320 \mathrm{~ms}$ & Frontal, Central & Temporal, Occipital \\
\hline $\mathrm{NTC}_{4}$ & $37 \cdot 41 \%$ & $400-700 \mathrm{~ms}$ & $676 \mathrm{~ms}$ & Right Central, Right Parietal, Occipital & Left Frontal, Left Temporal \\
\hline
\end{tabular}

Habituation, Experiment 1; $\mathrm{N}=$ Novelty detection, Experiment 2). Table 2 summarizes the TC characterization, including amount of total variance accounted for by each TC, latency range and latency at the peak of maximum variance, and topographic characterization by voltage (e.g., positive or negative deflections.

\subsubsection{Establishing patterns of mean amplitude change via multilevel models}

Statistical models were designed to test our two primary hypotheses: (1) whether ERP amplitude decreases with sequential stimulus presentation (Experiment 1), and (2) whether ERP amplitude to a novel speech sound also decreases across successive trials, while response amplitude to the already-familiar stimulus remains stable (Experiment 2). We also examined the spatial distribution of these changes in response amplitude across the scalp recording sites. For the statistical models, amplitude values were extracted as the average of all time points during the temporal window of the TC at the single trial level for each participant across five regions (Frontal, Central, Temporal, Parietal, and Occipital) and two hemispheres (Left, Right).

All preliminary unconditional models (see Supplemental Materials) supported a single-trial analytic approach by establishing that the majority of the variance in the data was accounted for by within-subject fluctuation across trials. As an overview, model selection procedures 
for each TC involved (a) first adding in fixed effects as a full-factorial design, and (b) sequentially removing non-significant higher-order interactions until the best fitting model was retained, based upon best practices guidelines (Hoffman, 2015). In this way, each TC multilevel model was permitted to consist of different fixed effects, which allowed for a deeper understanding of the factors that most influenced each TC, rather than including effects that did not contribute to the variance.

All multilevel models were estimated using PROC MIXED with restricted maximum likelihood in SAS 9.3, using the extracted amplitude values as the input data. Importantly, PROC MIXED and the maximum likelihood procedure is capable of accommodating unbalanced data (i.e., due to artifact rejection) such that missing trials do not contribute to the parameter estimates, ostensibly "skipping" any missing data. The presentation order of each trial was used as the metric of time to assess the trial-by-trial changes in amplitude (variable Trial Number). Trials within an experiment were centered at trial 1, such that the intercept is predicted for the start of the experiment. We elected this strategy because the beginning of each experiment is where the effects were expected to be most powerful (e.g. at the initial introduction of the novel speech syllable). Predictor variables were added as fixed effects to the full-factorial multilevel models (i.e., permitting all possible interactions), including Electrode Region, Electrode Hemisphere, Condition (only Experiment 2), and Trial Number. Both linear and quadratic effects of Trial Number were tested in order to account for the non-linear shape of habituation as predicted by theoretical models (Schöner \& Thelen, 2006). As described above, nonsignificant higher-order interactions were sequentially removed from the model in a systematic way to establish the best fitting model for each TC, per Hoffman, 2015. The omnibus tests for effects that contributed for each TC are reported in Table 3 and model parameter estimates are reported in Supplemental Materials. 
Table 3 Final model omnibus effects by temporal component (TC). Statistics are provided for the best fitting model (i.e., after removing non-significant fixed effects and higher-order interactions).

\begin{tabular}{|c|c|c|c|c|c|c|c|c|}
\hline \multicolumn{9}{|c|}{ Experiment 1: Habituation $(\mathrm{H})$} \\
\hline & \multicolumn{2}{|l|}{ HTC1 } & \multicolumn{2}{|l|}{ HTC2 } & \multicolumn{2}{|l|}{$\mathrm{HTC}_{3}$} & \multicolumn{2}{|l|}{$\mathrm{HTC}_{4}$} \\
\hline & $F$ & $p$ & $F$ & $p$ & $F$ & $p$ & $F$ & $p$ \\
\hline Region & 12.74 & $<0.0001$ & 0.0021 & 0.0021 & 4.24 & 0.002 & 12.92 & $<0.0001$ \\
\hline Hemisphere & & & & & & & 3.65 & 0.0561 \\
\hline Linear Trial Number & & & & & & & 1.53 & 0.2167 \\
\hline Hemisphere $\times$ Linear Trial & & & & & & & 6.9 & 0.0086 \\
\hline \multicolumn{9}{|c|}{ Experiment 2: Novelty detection $(\mathrm{N})$} \\
\hline & \multicolumn{2}{|l|}{ NTC 1} & \multicolumn{2}{|l|}{ NTC2 } & \multicolumn{2}{|c|}{$\mathrm{NTC}_{3}$} & \multicolumn{2}{|c|}{$\mathrm{NTC}_{4}$} \\
\hline & $F$ & $p$ & $F$ & $p$ & $F$ & $p$ & $F$ & $p$ \\
\hline Region & & & 11.22 & $<0.0001$ & 4.39 & 0.0015 & 15.61 & $<0.0001$ \\
\hline Condition & & & 11.09 & 0.0009 & & & 11.34 & 0.0008 \\
\hline Hemisphere & & & 9.16 & 0.0025 & 10.11 & 0.0015 & 14.5 & 0.0001 \\
\hline Linear Trial Number & & & 8.09 & 0.0045 & & & 8.88 & 0.0029 \\
\hline Quadratic Trial Number & & & 8.37 & 0.0038 & & & $7 \cdot 54$ & 0.0061 \\
\hline Condition $\times$ Hemisphere & & & 16.27 & $<0.0001$ & & & 18.62 & $<0.0001$ \\
\hline \multicolumn{9}{|l|}{ Interactions with Linear Trial } \\
\hline Linear $\times$ Condition & & & 7.27 & 0.007 & & & 5.6 & 0.018 \\
\hline Linear $\times$ Hemisphere & & & 12.16 & 0.0005 & & & 15.48 & $<0.0001$ \\
\hline Linear $\times$ Condition $\times$ Hem & phere & & 9.46 & 0.0021 & & & $9 \cdot 51$ & 0.0021 \\
\hline \multicolumn{9}{|c|}{ Interactions with Quadratic Trial } \\
\hline Quadratic $\times$ Condition & & & 7.23 & 0.0072 & & & 4.67 & 0.0307 \\
\hline Quadratic $\times$ Hemisphere & & & 12.05 & 0.0005 & & & $13 \cdot 32$ & 0.0003 \\
\hline Quadratic $\times$ Condition $\times \mathrm{H}$ & misphere & & 9.25 & 0.0024 & & & 8.39 & 0.0038 \\
\hline
\end{tabular}

\section{Results}

\subsection{Experiment 1: Habituation TC characterization overview}

See Table 2 for full characterization of Habituation Temporal Components (HTCs). All four HTCs accounted for a significant portion of variability. Early HTCs (before $300 \mathrm{~ms}$ ) included HTC1, which reflected an early positive posterior portion of the waveform, and HTC2, which was most evident as a negative-going transition over central and temporal electrodes. The mid-latency HTC3 elicited positivity across frontal and central electrodes, whereas the late latency $\mathrm{HTC}_{4}$ transitioned to negativity across frontal, central, and temporal electrodes. Grand average waveforms averaged across all electrode clusters are provided as Supplemental Fig. 1. 
3.2. Experiment 1: Habituation results.

3.2.1. HTC1 (4-12O ms)

In the final model for the early peak, only a significant effect of Electrode Region was retained. HTC1 mean amplitude was predicted to be positive (i.e., significantly greater than zero) across parietal and occipital electrode clusters and negative (i.e., significantly less than zero) across frontal and temporal electrode clusters.

\subsubsection{HTC2 (112-252 ms)}

The model for HTC2 followed a similar pattern to that of HTC1. Only a significant effect of Electrode Region was retained. HTC2 predicted mean amplitude to be significantly negative-going in the temporal electrode cluster.

\subsection{3. $\mathrm{HTC}_{3}$ (244-46o ms)}

The final model for $\mathrm{HTC}_{3}$ followed a similar pattern to that of the preceding components. Only a significant effect of Electrode Region was retained, indicating negative mean amplitude across temporal electrodes during this time window.

\subsubsection{HTC4 (404-70O ms)}

In the model for $\mathrm{HTC}_{4}$, an interaction between the linear effect of Trial Number and Electrode Hemisphere was retained, after controlling for the main effect of Electrode Region. This model indicated positive mean amplitude across parietal and occipital electrode clusters and negative mean amplitude across frontal and temporal electrode clusters. The amplitude of $\mathrm{HTC}_{4}$ was predicted to change significantly over sequential Trial Number by a linear trend toward zero for each hemisphere. In other words, at the first trial, mean amplitude was predicted to be positive for right hemisphere electrodes and negative for left hemisphere electrodes. Over the course of the experiment, the positive right hemisphere mean amplitude decreased and the negative left hemisphere mean amplitude increased, as illustrated in Fig. 1a. Fig. 1b illustrates the scalp distribution change over trials. 


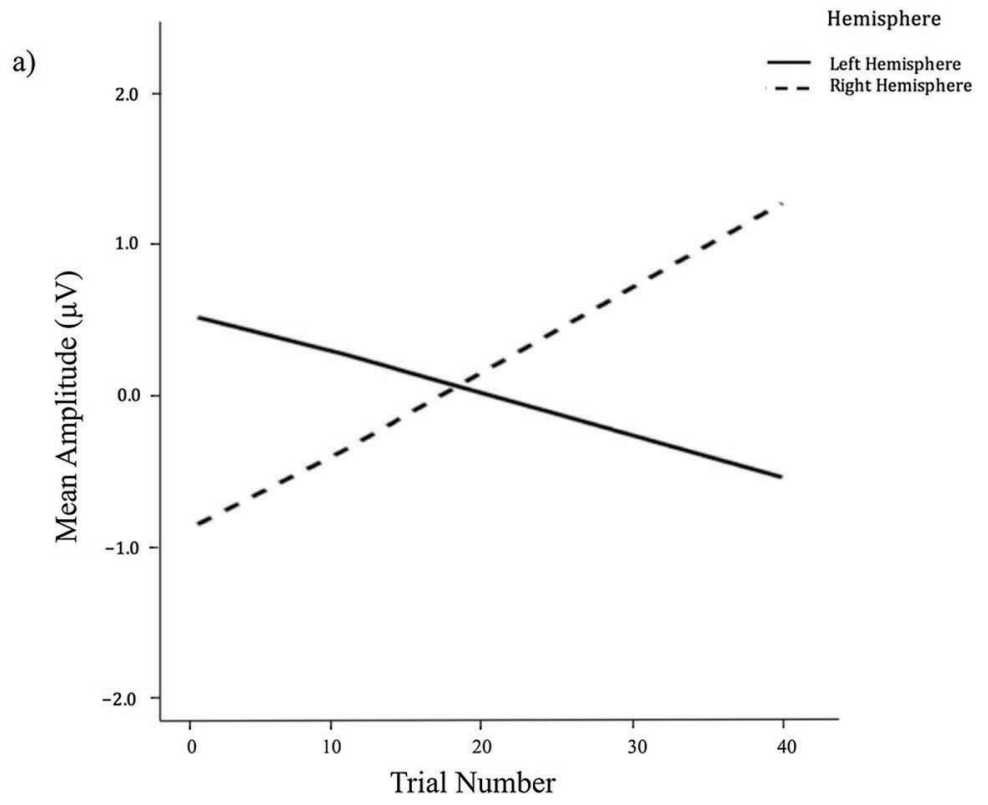

b)

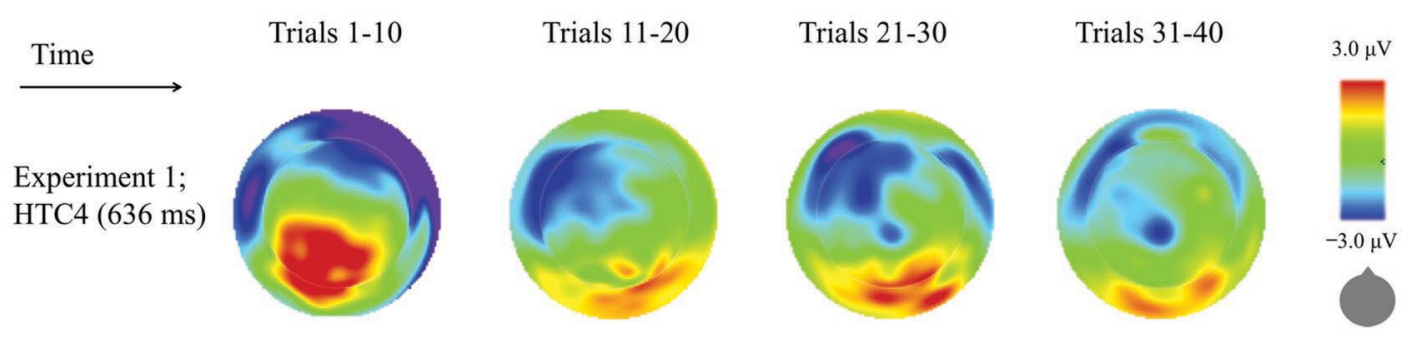

Fig. 1. Experiment 1 Effect of Trial Number. Figure a shows the linear effect of trial number for the $\mathrm{HTC}_{4}$ is drawn for each Electrode Hemisphere. Left hemisphere predicted mean amplitude values are drawn as a solid line, while right hemisphere values are drawn as dashed line. Model-predicted amplitude values increase/ decrease toward zero from trial 1 through approximately the midpoint of the test session, where they continue to diverge. Figure b shows scalp topographic maps illustrate the effect of Trial Number on $\mathrm{HTC}_{4}$ amplitude for groups of 10 trials (i.e., Trials 1-10 averaged for the left-most topographic plot). The pattern indicates that strong negative (i.e., blue) and positive (i.e., red) voltages increase and decrease, respectively, toward zero (i.e., green) over sequential trials. This figure illustrates the attenuation of newborn ERP response amplitude as a speech sound stimulus is repeated, and that this pattern occurs differently at scalp recording locations. 


\subsection{Experiment 1 summary}

Experiment 1 assessed whether newborns' brain responses to a repeated auditory stimulus changed in amplitude over 40 consecutive presentations. Habituation temporal component four (HTC4; 404-700 ms) accounted for a linear mean amplitude response decrement characteristic of habitation. The reduction in ERP mean amplitude occurred with opposing polarity for left and right hemisphere electrodes, consistent with prior literature describing early ERP laterality (i.e. Molfese \& Molfese, 1979). The linear function of the response decrement over successive trials lends itself to the interpretation that the decrement is a result of habituation rather than a refractory period of the neural generators (Picton, Hillyard, and Galambos, 1976). Next, in Experiment 2, we investigated infants' subsequent patterns of response recovery (i.e., orienting towards novel stimulus) during novelty detection by introducing a novel syllable in combination with the now habituated syllable.

\subsection{Experiment 2: Novelty detection characterization overview}

See Table 2 for full characterization of Novelty Temporal Components (NTCs) and illustration of grand-average waveforms in Fig. 2. Grand average waveforms averaged across all electrode clusters are provided as Supplemental Fig. 2. NTC1 did not account for sufficient maximal variance, thus was not included in further analyses. Based upon visual inspection of early latency NTC2 and late latency NTC4 (which are further clarified by statistical models below), there were different patterns for familiar and novel conditions, as well as differences between right and left hemispheres. Of note, both mid and late NTCs were similar in latency and topography to HTCs from Experiment 1.

\subsection{Experiment 2: Novelty detection results}

\subsubsection{NTC2 (4-232 ms)}

Fig. 3 illustrates the predicted mean amplitudes of NTC2 during Experiment 2. A significant main effect of Stimulus Novelty indicated that newborns detected the novel syllable as indexed by greater

mean amplitude to the novel than familiar syllable during the NTC2 

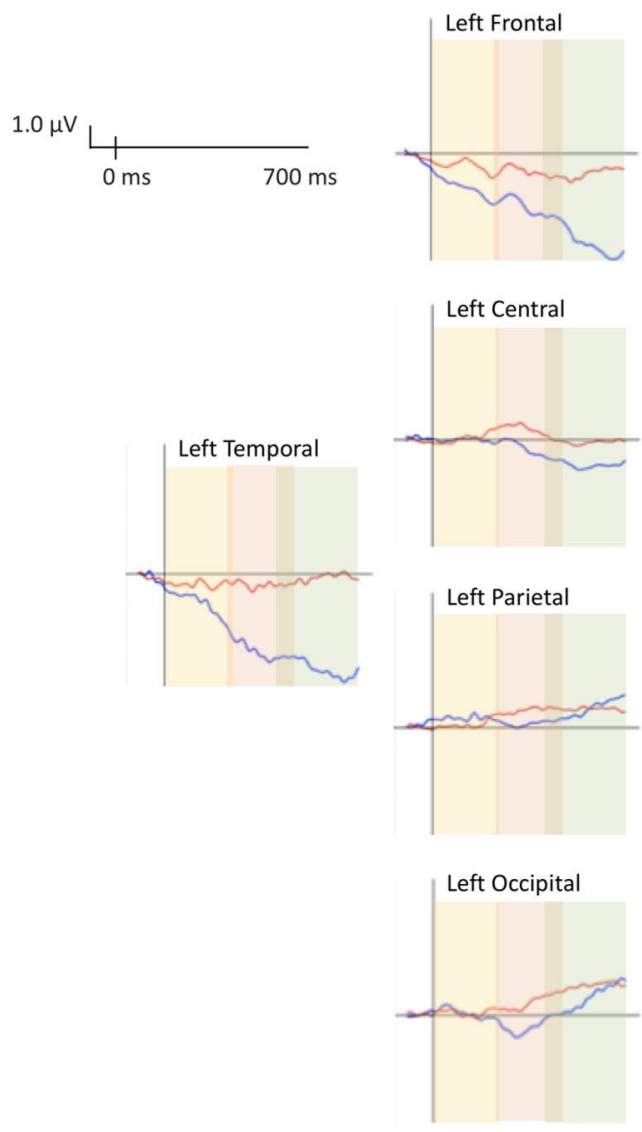
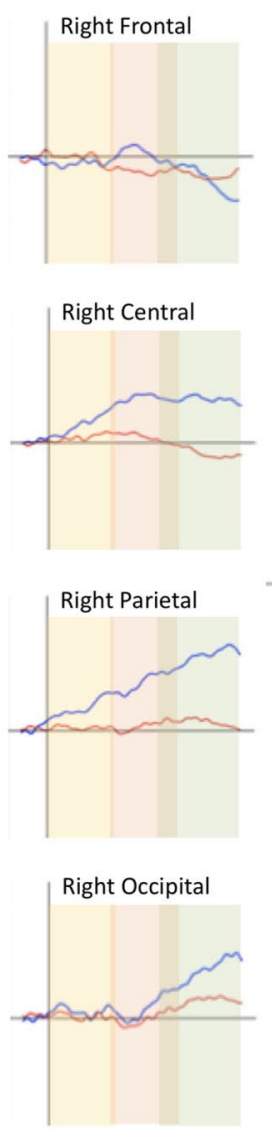

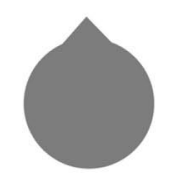

Condition

— Familiar

- Novel

\begin{tabular}{l|l|l|} 
NTC2 & NTC3 & NTC4 \\
\hline
\end{tabular}

Fig. 2. Experiment 2 Grand average waveforms by Electrode Cluster. Grand average waveforms for $\mathrm{N}=16$, drawn for each Electrode Cluster. Novel trials are drawn in red, while familiar trials are drawn in blue. The three Novelty Temporal Components (NTCs) are highlighted in yellow (NTC2: 4-232 ms), orange (NTC3: 224-476 ms), and green (NTC4: 400-700 ms) shaded regions. Differences between novel and familiar waveforms demonstrate that newborns differentiate between novel and familiar speech syllables within $700 \mathrm{~ms}$ of stimulus onset.

time window. Specifically, in the left hemisphere at trial one, the response to novel syllables was predicted to be $3.36 \mu \mathrm{V}$ greater, with a more positive amplitude, than for familiar trials.

The final model described a significant three-way interaction between Stimulus Novelty, Electrode Hemisphere, and quadratic time (i.e., a change in the linear slope of Trial Number), indicating that mean amplitude response attenuation occurred differently over left and right hemisphere electrodes. The effect of quadratic time was specific to novel syllables. Specifically, for novel syllables, the expected 

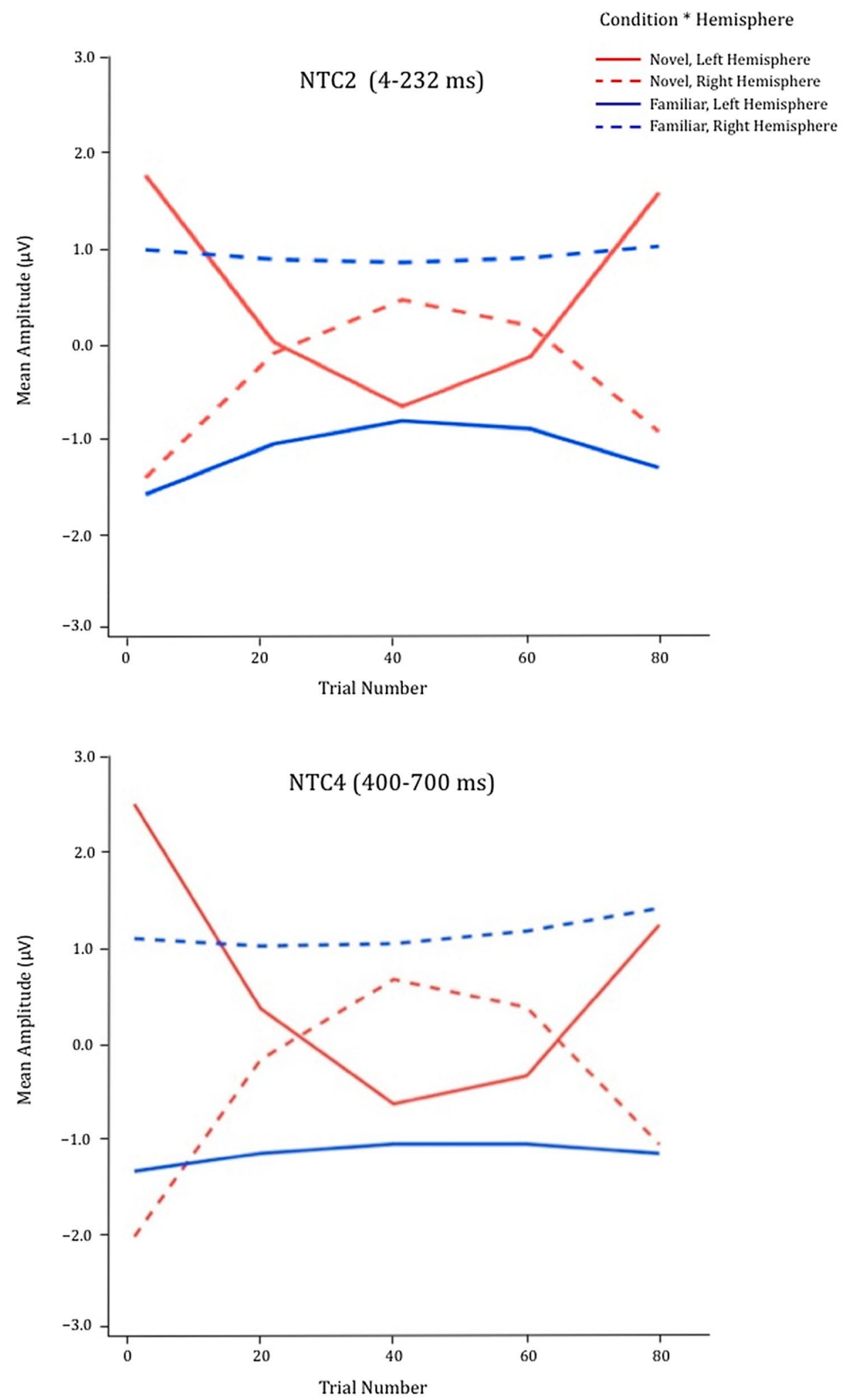
Fig. 3. Experiment 2 Model-estimated quadratic effect of Trial Number. The NTC2 and NTC4 mean component amplitudes are drawn for novel (red) and familiar (blue) trials, in each Electrode Hemisphere. Left hemisphere values are drawn in solid lines, and right hemisphere values in dashed lines. Non-linear trajectories specific to novel trials demonstrate that the newborn brain response to a novel speech syllable changes over 40 sequential presentations.

NTC2 mean left hemisphere amplitude at the start of Experiment 2 was $1.81 \mu \mathrm{V}$, with a significant expected instantaneous linear rate of decrease of $-0.12 \mu \mathrm{V}$ per trial. That linear rate of amplitude decrease was expected to slow, becoming significantly less negative by $0.003 \mu \mathrm{V}$ (twice the quadratic coefficient) per trial. A similar slowing quadratic effect, but with opposite polarity, was observed for novel trials over the right hemisphere electrodes. For right hemisphere electrode sites, novel trials were estimated to elicit negative NTC2 amplitudes of $-1.37 \mu \mathrm{V}$ at the start of Experiment $2(S E=0.74, p=.066)$, and become significantly less negative by $0.09 \mu \mathrm{V}$ per trial $(S E=$ 0.04, $p=.037)$. The linear rate of increase was expected to slow by $-0.002 \mu \mathrm{V}$ (computed as twice the quadratic coefficient) per trial $(S E=0.0005, p=.044)$. Notably, there were no trial-by-trial changes for Familiar trials.

The difference between Stimulus Novelty conditions became smaller over time, with a significant expected instantaneous linear rate of change of $0.16 \mu \mathrm{V}$ per trial at the start of Experiment 2, which slowed by $-0.004 \mu \mathrm{V}$ per trial. Thus, the differentiation between familiar and novel syllables was predicted to be largest at the start of the experiment, and decrease over time, by a slowing quadratic effect.

\subsection{2. $\mathrm{NTC}_{3}(224-476 \mathrm{~ms})$}

For NTC3, the best fitting model included only the main effects of Electrode Region and Electrode Hemisphere. NTC3 mean amplitude is predicted to be negative for left hemisphere electrodes, and significantly less negative for the right hemisphere. In addition, NTC3 mean amplitude is predicted to be significantly positive over frontal, central, and parietal Electrode Regions. o Stimulus Novelty or Trial Number effects for NTC3. 


\subsection{3. $\mathrm{NTC}_{4}$ (400-700 $\left.\mathrm{ms}\right)$}

A significant main effect of Stimulus Novelty indicated that newborns detected the novel syllable as indexed by a larger neural response to the novel than familiar syllable for the NTC4 mean amplitude at the start of Experiment 2. Specifically, for left hemisphere electrodes at trial one, the response to novel syllables was predicted to be $3.84 \mu \mathrm{V}$ greater, with more positive amplitude, compared to familiar syllables.

Fig. 3 illustrates the predicted mean amplitudes of $\mathrm{NTC}_{4}$ at four additional time points during Experiment 2. As shown, the final model described a significant three-way interaction between Stimulus Novelty, Electrode Hemisphere, and the quadratic effect of Trial Number, similar to that of the NTC2 in this experiment. As with NTC2, the trialby-trial mean amplitude change was specific to novel trials only and occurred differently in the left and right hemispheres for NTC4. Specifically, in the left hemisphere, novel trials were predicted to elicit positive NTC4 amplitudes of $2.51 \mu \mathrm{V}$, with an expected instantaneous linear rate of decrease of $-0.14 \mu \mathrm{V}$ per trial. That linear rate of decrease was expected to slow, becoming significantly less negative by $0.003 \mu \mathrm{V}$ (twice the quadratic coefficient) per trial. Again, the slowing quadratic effect was observed with opposite polarity in right hemisphere electrode sites. In the right hemisphere, novel trials were estimated to elicit negative NTC 4 amplitude of $-2.01 \mu \mathrm{V}$ at the start of Experiment 2, and become significantly less negative by $0.12 \mu \mathrm{V}$ per trial $(S E=0.05, p=.010)$. This increase is predicted to slow by a quadratic effect of $-0.003 \mu \mathrm{V}$ per trial $(S E=0.0006, p=$ .016). There were neither linear nor non-linear trial-by-trial changes for familiar trials.

The difference between conditions became smaller over time, with a significant expected instantaneous linear rate of change of $0.16 \mu \mathrm{V}$ per trial at the start of Experiment 2, which slowed by $-0.003 \mu \mathrm{V}$ per trial. Thus, the best fitting model predicted the amplitude difference between familiar and novel trials to be largest at the start of the experiment and decrease over time by a slowing quadratic effect.

\subsection{Experiment 2 summary}

Experiment 2 assessed whether newborns' brain response demonstrated response recovery to a novel syllable, as well as habituation 
to the initially novel syllable as it was repeated over many trials, in a similar manner as in Experiment 1. Both novelty temporal component (NTC) 2 (4-232 ms) and NTC4 (400-700 ms) exhibited a quadratic pattern of change for novel syllables (shown in Fig. 3). Fig. 3 illustrates the topographic distribution of activation across the scalp for these two components over time. This pattern was similar to, yet distinct from, Experiment 1 such that the rate of habituation to novel syllables slowed over time, following a quadratic trend rather than the linear trend observed in Experiment 1. This may be due to the methodological differences between Experiments 1 and 2; one repeated syllable versus equiprobable presentation of two different syllables, respectively. Similar to Experiment 1, these effects occurred with opposing polarity in the left and right hemispheres. There were no trial-by-trial changes for Familiar trials. These results indicate that newborns successfully difiliar from novel syllables, and that they dynamically enovelty over time.

\section{Discussion}

This study describes patterns of change in the dynamic trial-bytrial changes in the newborn ERP response that index habituation and novelty detection abilities shortly after birth. Consistent with known behavioral and neurophysiological literature, ERPs recorded during Experiment 1 indicate that newborns habituate to a repeated syllable because the ERP amplitude followed a linear trend toward zero over sequential trials. In addition, newborns successfully differentiated a novel from familiar syllable in Experiment 2. Quadratic change in amplitude specific to novel trials indicates dynamic encoding of the novel syllable during the task, in that the response changes systematically as exposure to the stimulus increases. These results provide evidence of habituation, novelty detection, and dynamic encoding of novel stimuli from 1 to 3 days of age and may serve as predictors of future language and speech.

Experiment 1 assessed newborn habituation and identified a linear change over the course of the experiment, such that ERP amplitude to a speech sound decreased over time. This indicates that the newborn's brain response steadily habituated to a repeated auditory stimulus over 40 repetitions. The lack of quadratic change (i.e., change in 
rate, as in slowing) may indicate that newborns are still actively encoding information about the repeated stimulus. ERP topography also shifted in topography over time (as evident in Fig. 1a), which may suggest a dipole shift (i.e., origination of the signal in brain source space) as habituation and learning occurred. It is possible that the newborn brain recruits different brain regions or shifts between neural networks as they habituate to a speech syllable. In addition, the modelpredicted amplitudes attenuated toward zero, which may suggest that newborns employ fewer neural resources to process the already-familiar auditory stimulus. These results support the stimulus comparator theory such that a familiar stimulus would induce a smaller orienting response after several presentations. In other words, dynamic learning occurs as newborns rapidly and successfully build a mental representation of the speech syllable. An alternative yet not mutually exclusive explanation may be that amplitude attenuation over time reflects infant fatigue. Future work is likely to benefit from paradigms designed to disentangle these alternative explanations.

Decreasing ERP amplitude during auditory habituation tasks has been shown in older infants (Chen, Peter, \& Burnham, 2016) and adults (Ritter, Vaughan, \& Costa, 1968). Some researchers acknowledge that habituation may index top-down processing, a most basic form of learning, or a prerequisite for learning within a test session (Puce, Allison, \& McCarthy, 1999; Rankin et al., 2009). This interpretation is similar to the stimulus-comparator model's conceptualization of behavioral habituation as information processing leading to the construction of a mental representation. An alternative explanation posits a frequency-based refractory response, but only if response decrements stabilize immediately with stimulus repetition (Budd, Barry, Gordin, Rennie, \& Michie, 1998). Evidence of response recovery to a novel stimulus provides some additional evidence for inference of habituation as a basic learning mechanism rather than simply the refractoriness of the neural substrate. Although inferences about habituation and novelty detection must be interpreted with caution, neural response attenuation which recovers to a novel stimulus may indicate an encoded memory of the familiar stimulus, consistent with the stimulus-comparator theory (Colombo \& Mitchell, 2009; Matuz et al., 2012). Indeed, an increase in neural response amplitude following exposure to initially novel pseudo words, has been interpreted 
to indicate the formation of neural memory traces in adults (Shtyrov, Nikulin, \& Pulvermüller, 2010).

In the present study, Experiment 2 introduced a novel syllable to identify mechanisms of novelty detection. Newborns demonstrated a greater orienting reflex in response to the novel syllable, as hypothesized. These results can be compared to the mismatch negativity (MMN) effect, in which a frequent (or standard) stimulus elicits a weaker response as compared to an infrequent (or deviant) stimulus (Čeponiene et al., 2002; Näätänen, 1990; 2001). While the MMN paradigm is somewhat similar in design to the novelty detection paradigm employed in Experiment 2, MMN designs confound stimulus frequency with stimulus familiarity, making it impossible to differentiate change detection from novelty detection in sensory memory. The equiprobable stimulus presentation in our Experiment 2 controls for stimulus frequency to a greater extent than the traditional MMN paradigm. However, the randomized order of stimuli creates four possible transitions across sequential stimuli (familiar-novel, novel-familiar, familiar-familiar, or novel-novel). It is possible that infants habituated to the familiar-familiar transition during Experiment 1, and responded differentially to the familiar-familiar transition compared to other transitions during Experiment 2. This interpretation requires that infants have both a representation of the stimulus identity as familiar or novel, as well as the learned transitional probabilities across stimuli. A large body of literature shows that the statistical probabilities across stimulus transitions can be detected by infants as well as adults, and propose it as a likely mechanism for the rapid acquisition of language (Bluf, Johnson, \& Valenza, 2011; Mittag, Takegata, \& Winkler, 2016; Teinonen, Fellman, Näätänen, Alku, \& Huotilainen, 2009). However, our study design included longer interstimulus intervals (i.e., 1100-1300 ms) than some (e.g., stimulus onset asynchrony of $135 \mathrm{~ms}$ in Mittag et al., 2016; $200 \mathrm{~ms}$ interstimulus interval in Teinonen, Fellman, Näätänen, Alku, \& Huotilainen, 2009), which may weaken the argument that infants are selectively learning certain kinds of transitions.

The differentiation between familiar and novel speech syllables was largest at the beginning of the experiment and decreased over time, by a slowing quadratic effect. As hypothesized, this may indicate that newborns devote fewer and fewer neural resources to processing 
those syllables for which they have already built a mental representation. By the midpoint of Experiment 2 (i.e., after 20 repetitions of the novel syllable and 60 total repetitions of the familiar syllable, including exposure during Experiment 1), the condition effect was no longer significant. This may indicate that the novel syllable was fully encoded by that point, and that both the familiar and novel syllables had equivalently strong mental representations. Of note, change over time was found for the novel trials, while the response to familiar trials was stable over time. Considering that response attenuation was conditiondependent, this suggests that the newborns' responses did not simply reflect fatigue. Consequently, the change over time must be task-specific and dynamic, indicating that the newborn brain adjusts mental representations of the syllable over time and exposure. This has important implications for the dynamic plasticity of newborn cognition and memory and the nature of how novelty is detected and encoded early in development.

As illustrated in Fig. 3, the overall strength of the novel response (drawn in red) is quite striking. Familiar and novel syllables contrast in response strength, such that initially, stronger responses (i.e., more positive and more negative) are predicted for novel compared to familiar syllables, especially for the late ERP components (e.g., NTC4). However, after approximately 10-15 trials, novel syllables elicit weaker (closer to zero) average amplitudes for the remainder of the experiment, indicating a smaller orienting response after sufficient exposure. This shift from initial novelty preference to a familiarity preference is consistent with behavioral research on infant looking time (Rose et al., 1982). However, it is possible that the shift from greater to lesser response strength for novel trials reflects an increase in response variability at the individual level. Increased response variability, once aggregated for the group, could create the effect of smaller amplitudes on average.

In addition, the initial decrease in response strength, as hypothesized, is followed by a subsequent increase in response strength for the second half of experiment 2, an unexpected finding. It is well established that infant familiarity and novelty preferences are not stable, and that they change dynamically as a consequence of age, amount of stimulus exposure, stimulus properties such as complexity, and stimulus presentation order (e.g. Rose et al., 1982; Schöner \& Thelen, 
2006). Research by Stets and Reid (2011) also finds infant ERP response strength shifts throughout an experiment as a function of stimulus exposure. Stets and Reid (2011) re-analyzed previously published ERP data from 4-month-old infants which investigated attentional differences between viewing images of an actor with object-directed gaze compared to averted gaze (Hoehl, Reid, Mooney, \& Striano, 2008). Original analyses of averaged ERP data for all artifact-free trials (between 11 and 37 trials included per participant, $\mathrm{n}=17$ ) found greater negative component amplitude for the averted gaze condition (Hoehl et al., 2008). Subsequent analyses which analyzed only a selection of trials from the start of the experiment found the opposite effect of greater negative component amplitude for the object-directed gaze condition (Stets \& Reid, 2011). This type of finding confirms that the interpretation of infant cognition by measuring stimulus differentiation is quite complex and subject to dynamic shifts.

As the increase in response strength to novel stimuli in the second half of experiment 2 was an unexpected finding, we do not make strong interpretations for why it may have occurred. It may be the case that while the quadratic model was the best fitting model of those we tested (i.e., polynomial functions), it is possible other nonlinear models would provide a more accurate portrayal of the data. For instance, whereas polynomial functions such as quadratic change are often criticized for predicting a return or reverse to the original values, exponential models may better capture how changes over time eventually establish a baseline or plateau (Hoffman, 2015). However, by examining infant ERP data on a trial-by-trial level as in the present study, this study represents a first step toward demonstrating the underlying dynamic shifts in familiarity and novelty preferences.

One outstanding question is whether or not the components analyzed in this study reflect true newborn brain signal or other, uncharacterized activity - including experimental artifacts (i.e., amplifier or sensor net), environmental noise, or perhaps biological artifacts (i.e., gustatory response). An advantage of the statistical strategy is that random effects models account for individual variability, such that the covariance matrix adjusts for each trial. In this way, we reduce the likelihood of artifacts over-contributing to the model under the assumption that these effects are fairly stable throughout the duration of the experiment. While this may not account for other biological 
factors, our results are similar to other work with newborns (e.g., Leppänen et al., 2010) suggesting that the components reflected cognitive processes, rather than biological processes more broadly.

Limitations of the present study include the relatively small sample size, which did not permit the estimation of random subject effects in the multilevel models. Although our trial-by-trial approach provides the opportunity to address signal change over time, additional work should address individual differences in response variability over time. Future research should examine the individual differences in infants' neural habituation as well as detection and encoding of novelty. In addition, all participants in the present study were healthy newborns with no family history of learning or language disorders. The developmental outcomes of these infants are unknown, and it is possible that increased variation in individual newborn ERPs is related to later developmental learning or language problems.

Lastly, it is possible that our selected filter setting adversely influenced the resulting brain waves that served to determine the temporal windows of interest. For instance, Weber, Hahne, Friedrich, and Friederici (2004) found a mismatch negativity component observed in 4-5-month-old infants with filter settings of a 1-15 Hz bandpass, but not a $0.3 \mathrm{~Hz}$ highpass filter. Of particular concern would be distortion of the data such that problematic filter settings cause artifacts that either augment peak components, shift peak latencies, or create artifactual inverted peaks on either side of a peak component. Although to our knowledge no studies have systematically tested for these problems in neonates, work in adults indicates that high-pass filters above $0.3 \mathrm{~Hz}$ may induce artifactual components at the ERP level (Tanner, MorganShort, \& Luck, 2015). Here, our filter settings were limited by the software package; however, future work should consider the extent by which these processing decisions affect the data, especially given the tPCA approach for temporal window selection.

These results have important implications for understanding the neurodevelopmental underpinnings of language in early infancy. Newborn ERPs have been shown to predict developmental risk or outcomes including language ability and dyslexia, based on the differentiation between phonemic contrasts (Fellman et al., 2004; Guttorm et al., 2010; Leppänen et al., 2012; Molfese, 2000; Thiede et al., 2019). However, trial-by-trial analysis methods may provide 
additional sensitivity to detect developmental risk in multiple domains (e.g., memory, attention) in addition to language. For example, speed of neural habituation may provide an indication of the efficiency of a newborn's neural network, while novelty detection, or the degree of differentiation between familiar and novel stimuli, may indicate risk for future cognitive disability.

\subsection{Conclusions}

The present study finds that newborn ERP amplitudes attenuate over sequential trials in response to repeated speech syllables, indexing habituation. In addition, newborns successfully differentiated a novel from familiar syllable at the start of a novelty detection task. Newborns also habituated to the novel stimulus as exposure increased, but did not further habituate to the already-familiar stimulus. These results provide evidence of habituation, novelty detection, and dynamic encoding of novel stimuli from 1 to 3 days of age.

These mechanisms set up important opportunities for learning in early infancy.

Author contributions: CC participated in the data collection, conceived and performed the statistical analyses, and drafted the manuscript; $\mathrm{CH}$ participated in the conception and design of the study, data collection, and helped to draft the manuscript; DM conceived of the study, and participated in its design and coordination, and provided edits to the manuscript.

Statement of significance to the neurobiology of language: This study represents basic science examining language mechanisms at birth. We use electrophysiology to assess habituation to a repeated speech syllable as well as the detection of a novel syllable, significant for understanding the neurobiology of language, specifically the neural underpinnings of language sound processing soon after birth.

Competing Interests and Funding: None. This research did not receive any specific grant from funding agencies in the public, commercial, or not-for-profit sectors.

Appendix A. Supplementary material: Supplementary data follow the References; two additional spreadsheets are attached to the html record for this article. 


\section{References}

Amsel, B. (2011). Tracking real-time neural activation of conceptual knowledge using single-trial event-related potentials. Neuropsychologia, 49, 970983. https://doi.org/ 10.1016/j.neuropsychologia.2011.01.003.

Bender, S., Banaschewski, T., Roessner, V., Klein, C., Rietschel, M., Feige, B., ... Laucht, M. (2015). Variability of single trial brain activation predicts fluctuations in reaction time. Biological Psychology, 106, 50-60. doi:10.1016/j. biopsycho.2015.01.013.

Blankertz, B., Lemm, S., Treder, M., Haufe, S., \& Muller, K. (2011). Single-trial analysis and classification of ERP components - A tutorial. NeuroImage, 56, 814-825.

Bluf, H., Johnson, S. P., \& Valenza, E. (2011). Visual statistical learning in the newborn infant. Cognition, 121, 127-132. doi:10.1016/j.cognition.2011.06.010.

Bridger, W. H. (1961). Sensory habituation and discrimination in the human neonate. American Journal of Psychiatry, 117, 991-996.

Budd, T. W., Barry, R. J., Gordin, E., Rennie, C., \& Michie, P. T. (1998). Decrement of the N1 auditory event-related potential with stimulus repetition: Habituation vs. refractoriness. International Journal of Psychophysiology, 31, 51-68.

Carral, V., Huotilainen, M., Ruusuvirta, T., Fellman, V., Näätänen, R., \& Escera, C. (2005). A kind of auditory 'primitive intelligence' already present at birth. European Journal of Neuroscience, 21, 3201-3204. doi:10.1111/j.1460-9568.2005.04144.x.

Cattell, R. B. (1966). The scree test for the number of factors. Multivariate Behavioral Research, 1, 245-276.

Čeponiene, R., Kushnerenko, E., Fellman, V., Renlund, M., Suominen, K., \& Näätänen, R. (2002). Event-related potential features indexing central auditory discrimination by newborns. Cognitive Brain Research, 13, 101-113.

Chen, A., Peter, V., \& Burnham, D. (2016). Auditory ERP response to successive stimuli in infancy. PeerJ, 4, e1580. doi:10.7717/peerj.1580.

Colombo, J., \& Mitchell, D. W. (2009). Infant visual habituation. Neurobiology of Learning and Memory, 92, 225-234. doi:10.1016/j.nlm.2008.06.002.

Colombo, J., Mitchell, D. W., Coldren, J. T., \& Freeseman, L. J. (1991). Individual dif-ferences in infant visual attention: Are short lookers faster processors or feature processors? Child Development, 62, 1247-1257.

Csibra, G., Kushnerenko, E., \& Grossmann, T. (2008). Electrophysiological methods in studying infant cognitive development. In C. A. Nelson, \& M. W. Collins (Vol. Eds.), Handbook of developmental cognitive neuroscience: Vol. 2, (pp. 247-262). Cambridge, MA: MIT Press.

Curran, T. (1999). The electrophysiology of incidental and intentional retrieval: ERP old/ new effects in lexical decision and recognition memory. Neuropsychologia, 37, 771-785.

Donchin, E. (1966). A multivariate approach to the analysis of average evoked potentials. IEEE Transactions on Biomedical Engineering, 3, 131-139. 
Fantz, R. L. (1964). Visual experience in infants: Decreased attention to familiar patterns relative to novel ones. Science, 146, 668-670.

Fellman, V., Kushnerenko, E., Mikkola, K., Čeponiene, R., Leipala, J., \& Näätänen, R. (2004). Atypical auditory event-related potentials in preterm infants during the first year of life: A possible sign of cognitive dysfunction? Pediatric Research, 56, 291-297. doi:10.1203/01.PDR.0000132750.97066.

Ferree, T. C. (2000). Spline interpolation of the scalp EEG. Secondary TitlEGI. Gonzalez-Frankenberger, B., Harmony, T., Ricardo-Garcell, J., PorrasKattz, E., Fernandez-Bouzas, A., Santiago, E., \& Avecilla-Ramirez, G. (2008). Habituation of visual evoked potentials in healthy infants and in infants with periventricular leukomalacia. Clinical Neurophysiology, 119, 2879-2886. doi:10.1016/j. clinph.2008.08.020.

Groves, P. M., \& Thompson, R. F. (1970). Habituation: A dual-process theory. Psychological Review, 77, 419-450.

Guttorm, T. K., Leppänen, P. H., Hamalainen, J. A., Eklund, K. M., \& Lyytinen, H. J. (2010). Newborn event-related potentials predict poorer pre-reading skills in children at risk for dyslexia. Journal of Learning Disabilities, 43, 391-401.

Hoehl, S., Reid, V., Mooney, J., \& Striano, T. (2008). What are you looking at? Infants' neural processing of an adult's object directed eye gaze. Developmental Science, 11(1), 10-16. doi:10.1111/j.1467-7687.2007.00643.x.

Hofmann, M. J., Salapatek, P., \& Kuskowski, M. (1981). Evidence for visual memory in the averaged and single evoked potentials of human infants. Infant Behavior and Development, 4, 401-421.

Hoffman, L. (2015). Longitudinal analysis: Modeling within-person fluctuation and change. New York, NY: Routledge.

Hoffman, L. (2007). Multilevel models for examining individual differences in within- person variation and covariation over time. Multivariate Behavioral Research, 42, 609-629.

Hoffman, L., \& Stawski, R. S. (2009). Persons as contexts: Evaluating betweenperson and within-person effects in longitudinal analysis. Research in Human Development, 6, 97-120. doi:10.1080/15427600902911189.

Houston-Price, C., \& Nakai, S. (2004). Distinguishing novelty and familiarity effects in infant preference procedures. Infant and Child Development: An International Journal of Research and Practice, 13, 341-348. doi:10.1002/ icd.364.

Hudac, C. M., Cortesa, C. S., Ledwidge, P. S., \& Molfese, D. L. (2018). History of concussion impacts electrophysiological correlates of working memory. International Journal of Psychophysiology, 132(Pt A), 135144. doi:10.1016/j.ijpsycho. 2017.09.020.

Hudac, C. M., DesChamps, T. D., Arnett, A. B., Cairney, B. E., Ma, R., Webb, S. J., \& Bernier, R. A. (2018). Early enhanced processing and delayed habituation to deviance sounds in autism spectrum disorder. Brain and Cognition, 123, 110-119.

Hunter, M. A., \& Ames, E. W. (1988). A multifactor model of infant preferences for novel and familiar stimuli. Advances in Infancy Research, 5, 69-95. 
Hunter, M. A., Ames, E. W., \& Koopman, R. (1983). Effects of stimulus complexity and familiarization time on infant preferences for novel and familiar stimuli. Developmental Psychology, 19, 338-352.

Johnson, M. H., de Haan, M., Oliver, A., Smith, W., Hatzakis, H., Tucker, L. A., \& Csibra, G. (2001). Recording and analyzing high-density event-related potentials with infants using the geodesic sensor net. Developmental Neuropsychology, 19, 295-323.

Kavšek, M. (2013). The comparator model of infant visual habituation and dishabituation: Recent insights. Developmental Psychobiology, 55, 793-808. https://doi. org/10.1002/dev.21081.

Kayser, J., \& Tenke, C. E. (2003). Optimizing PCA methodology for ERP component identification and measurement: Theoretical rationale and empirical evaluation. Clinical Neurophysiology, 114(12), 2307-2325. doi:10.1016/ S1388-2457(03)00241-4.

Key, A. P., Ferguson, M., Molfese, D. L., Peach, K., Lehman, C., \& Molfese, V. J. (2007). Smoking during pregnancy affects speech-processing ability in newborn infants. Environmental Health Perspectives, 115, 623-629.

Kushnerenko, E., Čeponiene, R., Balan, P., Fellman, V., \& Näätänen, R. (2002). Maturation of the auditory change detection response in infants: A longitudinal ERP study. NeuroReport, 13(15), 1843-1848.

Kushnerenko, E. V., Van den Bergh, B. R., \& Winkler, I. (2013). Separating acoustic deviance from novelty during the first year of life: A review of eventrelated potential evidence. Frontiers in Psychology, 4, 595. doi:10.3389/ fpsyg.2013.00595.

Leppänen, P. H., Hämäläinen, J. A., Guttorm, T. K., Eklund, K. M., Salminen, H., Tanskanen, A., ... Lyytinen, H. (2012). Infant brain responses associated with readingrelated skills before school and at school age. Clinical Neurophysiology, 42, 35-41. doi:10.1016/j.neucli.2011.08.005.

Leppänen, P. H., Hämäläinen, J. A., Salminen, H. K., Eklund, K. M., Guttorm, T. K., Lohvansuu, K., ... Lyytinen, H. (2010). Newborn brain event-related potentials re- vealing atypical processing of sound frequency and the subsequent association with later literacy skills in children with familial dyslexia. Cortex, 46(10), 1362-1376.

Little, V. M., Thomas, D. G., \& Letterman, M. R. (1999). Single-trial analyses of devel- opmental trends in infant auditory event-related potentials. Developmental Neuropsychology, 16, 455-478.

Mahmoudzadeha, M., Dehaene-Lambertzb, G., Fourniera, M., Kongoloa, G., Goudjila, S., Duboisb, J., ... Walloisa, F. (2013). Syllabic discrimination in premature human in- fants prior to complete formation of cortical layers. Proceedings of the National Academy of Sciences of the United States of America, 110, 4846-4851.

Matuz, T., Govindan, R. B., Preissl, H., Siegel, E. R., Muenssinger, J., Murphy, P., ... Eswaran, H. (2012). Habituation of visual evoked responses in neonates and fetuses: A MEG study. Developmental Cognitive Neuroscience, 2, 303316. doi:10. 1016/j.den.2012.03.001. 
Megela, A. L., \& Teyler, T. J. (1979). Habituation of the human evoked potential. Journal of Comparative and Physiological Psychology, 93, 1154-1170.

Milne, E. (2011). Increased intra-participant variability in children with autistic spectrum disorders: Evidence from single-trial analysis of evoked EEG. Frontiers in Psychology, 2, 51. doi:10.3389/fpsyg.2011.00051.

Mittag, M., Takegata, R., \& Winkler, I. (2016). Transitional probabilities are prioritized over stimulus/pattern probabilities in auditory deviance detection: Memory basis for predictive sound processing. Journal of Neuroscience, 36(37), 9572-9579. https://doi. org/10.1523/JNEUROSCI.1041-16.2016.

Molfese, D. L. (2000). Predicting dyslexia at 8 years of age using neonatal brain responses. Brain and Language, 72, 238-245. doi:10.1006/brln.2000.2287.

Molfese, D. L., \& Molfese, V. J. (1979). Hemisphere and stimulus differences as reflected in the cortical responses of newborn infants to speech stimuli. Developmental Psychology, 15, 505-511.

Molfese, V. J., Molfese, D. L., \& Modgline, A. A. (2001). Newborn and preschool pre- dictors of second-grade reading scores. Journal of Learning Disabilities, 34, 545-554. Molfese, D. L., Nunez, V., Seibert, S. M., \& Ramanaiah, N. V. (1976). Cerebral asymmetry: Changes in factors affecting its development. Annals of the New York Academy of Sciences, 280(1), 821-833.

Molfese, D. L., Tan, A., Sarkari, S., \& Gill, L. A. (1997). Prediction and intervention of school age language problems using electrophysiological measures obtained at birth. In S. M. Clancy Dollinger, \& L. DiLalla (Eds.). Asses and interv across the lifesp. Mahwah, NJ: Lawrence Erlbaum Associates.

Muenssinger, J., Matuz, T., Schleger, F., Kiefer-Schmidt, I., Goelz, R., WackerGussmann, A., ... Preissl, H. (2013). Auditory habituation in the fetus and neonate: An fMEG study. Developmental Science, 16, 287-295. doi:10.1111/ desc.12025.

Näätänen, R. (1990). The role of attention in auditory information processing as revealed by event-related potentials and other brain measures of cognitive function. Behavioral and Brain Sciences, 13, 201-288.

Näätänen, R. (2001). The perception of speech sounds by the human brain as reflected by the mismatch negativity $(\mathrm{MMN})$ and its magnetic equivalent (MMNm). Psychophysiology, 38, 1-21.

Nizami, L. (2019a). Too resilient for anyone's good: "Infant psychophysics" viewed through second-order cybernetics, part 1 (background and problems). Kybernetes, 48(4), 751-768. doi:10.1108/K-11-2017-0451.

Nizami, L. (2019b). Too resilient for anyone's good: "Infant psychophysics” viewed through second-order cybernetics, part 2 (re-interpretation). Kybernetes, 48(4), 769-781. doi:10.1108/K-05-2018-0238.

Nikkel, L., \& Karrer, R. (1994). Differential effects of experience on the ERP and behavior of 6-month-old infants: Trends during repeated stimulus presentations. Developmental Neuropsychology, 10, 1-11.

Oakes, L. M. (2010). Using habituation of looking time to assess mental processes in infancy. Journal of Cognition and Development, 11, 255-268. doi:10.1080/ 15248371003699977. 
Perani, D., Saccuman, M. C., Scifo, P., Anwander, A., Spada, D., Baldoli, C., ... Friederici, A. D. (2011). Neural language networks at birth. Proceedings of the National Academy of Sciences of the United States of America, 108, 1605616061. doi:10. 1073/pnas.1116o61108.

Picton, T. W., Hillyard, S. A., \& Galambos, R. (1976). Habituation and attention in the auditory system. In W. D. Keidel \& W. D. Neff (Eds.) Handbook of sensory physiology (Vol 3. pp. 343-390).

Picton, T. W., \& Taylor, M. J. (2007). Electrophysiological evaluation of human brain development. Developmental Neuropsychology, 31, 249-278.

Puce, A., Allison, T., \& McCarthy, G. (1999). Electrophysiological studies of human face perception. III: Effects of top-down processing on face-specific potentials. Cerebral Cortex, 9, 445-458.

Quinn, P. C., Yahr, J., Kuhn, A., Slater, A. M., \& Pascalis, O. (2002). Representation of the gender of human faces by infants: A preference for female. Percept, 31, 1109-1121.

Rankin, C. H., Abrams, T., Barry, R. J., Bhatnagar, S., Clayton, D. F., Colombo, J., ... Thompson, R. F. (2009). Habituation revisited: An updated and revised description of the behavioral characteristics of habituation. Neurobiology of Learning and Memory, 92(2), 135-138. doi:10.1016/j.nlm.2008.09.012.

Ritter, W., Vaughan, H. G., Jr., \& Costa, L. D. (1968). Orienting and habituation to short term changes in averaged evoked responses. $y, 25,550-556$.

Regtvoort, A. G., van Leeuwen, T. H., Stoel, R. D., \& van der Leij, A. (2006). Efficiency of visual information processing in children at-risk for dyslexia: Habituation of singletrial ERPs. Brain and Language, 98, 319-331. doi:10.1016/j. bandl.2006. 06.006.

Roder, B. J., Bushnell, E. W., \& Sasseville, A. M. (2000). Infants' preferences for familiarity and novelty during the course of visual processing. Infancy, 1, 491-507.

Rosburg, T., \& Sörös, P. (2016). The response decrease of auditory evoked potentials by repeated stimulation-Is there evidence for an interplay between habituation and sensitization? Clinical Neurophysiology, 127, 397-408.

Rose, S. A., Gottfried, A. W., Melloy-Carminar, P., \& Bridger, W. H. (1982). Familiarity and novelty preferences in infant recognition memory: Implications for information processing. Developmental Psychology, 18, 704-713.

Ruusuvirta, T., Huotilainen, M., Fellman, V., \& Näätänen, R. (2004). Newborn human brain identifies repeated auditory feature conjunctions of low sequential probability. European Journal of Neuroscience, 20, 2819-2821. doi:10.1111/j.1460-9568.2004.03734.x.

Schöner, G., \& Thelen, E. (2006). Using dynamic field theory to rethink infant habituation. Psychological Review, 113, 273-299. doi:10.1037/0033-295X.113.2.273.

Shtyrov, Y., Nikulin, V. V., \& Pulvermüller, F. (2010). Rapid cortical plasticity underlying novel word learning. Journal of Neuroscience, 30(50), 16864-16867. 
Snyder, K. A., Garza, J., Zolot, L., \& Kresse, A. (2010). Electrophysiological signals of familiarity and recency in the infant brain. Infancy, 15, 487-516. doi:10. 1111/j.1532-7078.2009.00021.x.

Snyder, K., Webb, S. J., \& Nelson, C. A. (2002). Theoretical and methodological im- plications of variability in infant brain response during a recognition memory para- digm. Infant Behavior and Development, 25, 466-494.

Sokolov, E. N. (1963a). Higher nervous functions: The orienting reflex. Annual Review of Physiology, 25, 545-580.

Sokolov, E. N. (1963b). Perception and the conditioned reflex. New York: MacMillan.

Sokolov, E. N. (1966). Orienting reflex as information regulator. In A. Leontyev, A. Luria, \& A. Smirnov (Eds.). Psychological research in U.S.S.R (pp. 334-360). Moscow: Progress Publishers.

Stets, M., \& Reid, V. M. (2011). Infant ERP amplitudes change over the course of an experimental session: Implications for cognitive processes and methodology. Brain and Development, 33(7), 558-568.

Stevens, K. N., \& Blumstein, S. E. (1978). Invariant cues for place of articulation in stop consonants. Journal of the Acoustical Society of America, 64, 1358-1368.

Streri, A., Hevia, M., Izard, V., \& Coubart, A. (2013). What do we know about neonatal cognition? Behavioral Science, 3, 154-169. doi:10.339o/ bs3010154.

Swain, I. U., Zelazo, P. R., \& Clifton, R. K. (1993). Newborn infants' memory for speech sounds retained over 24 hours. Developmental Psychology, 2, 312-323.

Tanner, D., Morgan-Short, K., \& Luck, S. J. (2015). How inappropriate high-pass filters can produce artifactual effects and incorrect conclusions in ERP studies of language and cognition. Psychophysiology, 52(8), 997-1009. doi:10.1111/ psyp. 12437.

Teinonen, T., Fellman, V., Näätänen, R., Alku, P., \& Huotilainen, M. (2009). Statistical language learning in neonates revealed by event-related brain potentials. BMC Neuroscience, 10, 21. doi:10.1186/1471-2202-10-21.

Thiede, A., Virtala, P., Ala-Kurikka, I., Partanen, E., Huotilainen, M., Mikkola, K., ... Kujala, T. (2019). An extensive pattern of atypical neural speech-sound discrimination in newborns at risk for dyslexia. Clinical Neurophysiology, 130, 634-646. https:// doi.org/10.1016/j.clinph.2019.01.019.

Thomas, D. G., Shucard, D. W., Shucard, J. L., \& Campos, J. J. (1989). Effects of stimulus and laboratory experience on auditory evoked potentials in infancy. Developmental Neuropsychology, 5, 25-32. doi:10.1080/87565648909540420.

Thompson, R. F., \& Spencer, W. D. (1966). Habituation: A model phenomenon for the study of neuronal substrates of behavior. Psychological Review, 73, 16-43.

Tremblay, A., \& Newman, A. J. (2015). Modeling nonlinear relationships in ERP data using mixed-effects regression with R examples. Psychophysiology, 52, 124-139. doi:10.1111/psyp.12299. 
Tsao, F., Liu, H., \& Kuhl, P. K. (2004). Speech perception in infancy predicts language development in the second year of life: A longitudinal study. Child Development, 75, 1067-1084.

Turk-Browne, N. B., Scholl, B. J., \& Chun, M. M. (2008). Babies and brains: Habituation in infant cognition and functional neuroimaging. Frontiers in Human Neuroscience, 2, 16. doi:10.3389/neuro.09.016.2008.

Wastell, D. G., \& Kleinman, D. (1980a). Fast habituation of the late components of the visual evoked potential in man. Physiology \& Behavior, 25, 93-97.

Wastell, D. G., \& Kleinman, D. (1980b). Potentiation of the habituation of human brain potentials. Biological Psychology, 10, 21-29.

Weber, C., Hahne, A., Friedrich, M., \& Friederici, A. D. (2004). Discrimination of word stress in early infant perception: Electrophysiological evidence. Cognitive Brain Research, 18(2), 149-161.

Wichary, S., Magnuski, M., Oleksy, T., \& Brzezicka, A. (2014). Neural signatures of rational and heuristic choice strategies: A single trial ERP analysis. Frontiers in Human Neuroscience, 11, 401. doi:10.3389/fnhum.2017.00401.

Wiebe, S. A., Cheatham, C. L., Lukowski, A. F., Haight, J. C., Muehleck, A. J., \& Bauer, P. J. (2006). Infants' ERP responses to novel and familiar stimuli change over time: Implications for novelty detection and memory. Infancy, 9, 21-44.

Wolfe, C. D., \& Bell, M. A. (2007). The integration of cognition and emotion during in-ulatory processes associated with the development of. Brain and Cognition, 65, 3-13. 


\section{$\underline{\text { Supplemental Materials I: Electrode clusters used for ERP analysis }}$}

Electrode numbers for the EGI 128 Geodesic Sensor Net Electrode layout divided into 10 scalp regions. Electrode numbers from the $128 \mathrm{GSN}$ for each region are listed below.

Left Frontal: 18, 19, 21, 22, 23, 24, 25, 26, 27, 32, 33, 38

Right Frontal: 1, 2, 3, 4, 8, 9, 10, 14, 121, 122, 123, 124

Left Central: 7, 12, 13, 20, 28, 29, 30, 31, 34, 35, 36, 37, 40, 41, 42, 46, 47

Right Central: 5, 80, 87, 93, 98, 102, 105, 106, 109, 110, 111, 112, 116, 117, 118

Left Temporal: 39, 43, 44, 45, 48, 49, 56

Right Temporal 107, 108, 113, 114, 115, 119, 120

Left Parietal: 50, 51, 52, 53, 54, 57, 58, 59, 60, 61, 67

Right Parietal: 77, 78, 79, 85, 86, 91, 92, 96, 97, 100, 101

Left Occipital: 63, 64, 65, 66, 68, 69, 70, 71, 73, 74

Right Occipital: 76, 82, 83, 84, 88, 90, 94, 95, 99

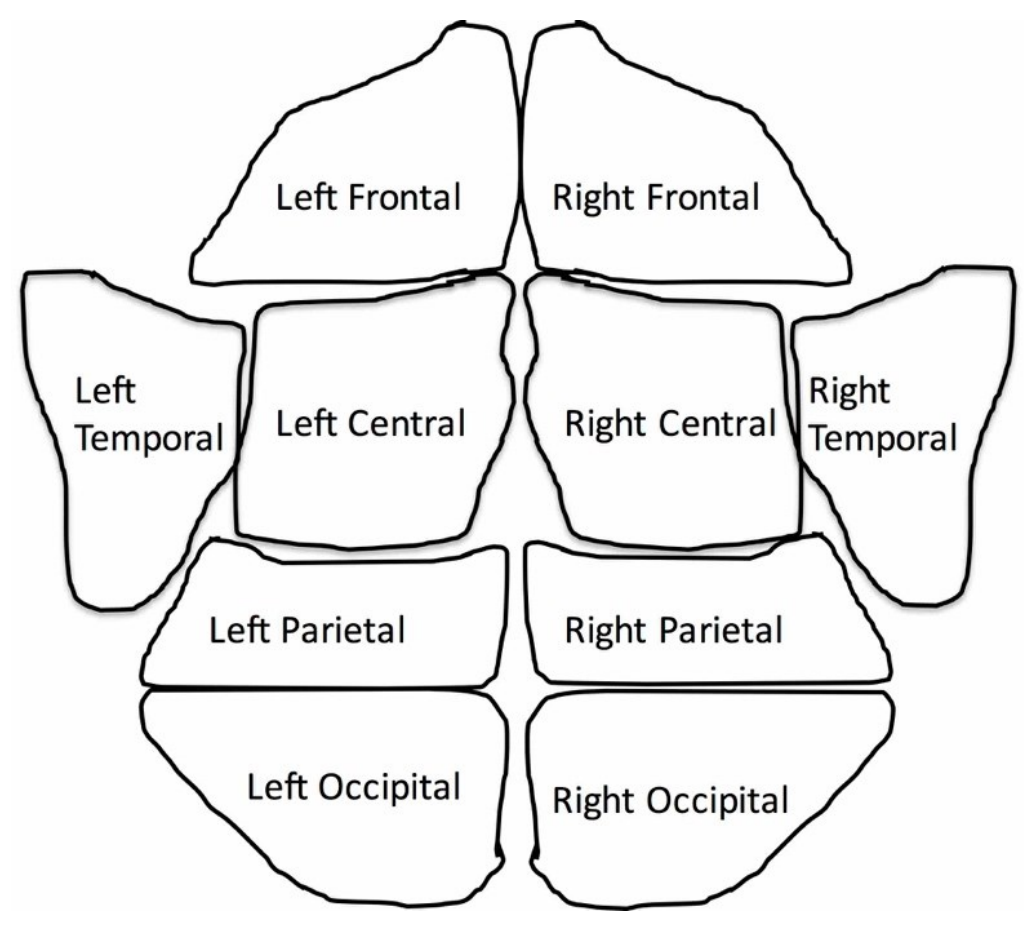




\section{$\underline{\text { Supplemental Materials II: Unconditional mixed multilevel models }}$}

\section{Experiment 1}

Unconditional Models: The intraclass correlation for each of the temporal components rounded to an equivalent -0.08 . Negative intraclass correlations can be understood as zero reliability among individuals (Bartko, 1976, Aarts et al., 2014). In other words, withinsubject difference accounted for a great majority of total variance, or that each observation (trial) obtained from a single participant are independent. Therefore, a great majority of the variance in the data was accounted for by within-subject fluctuation across trials, as compared to between-subject differences on average. This supported the novel analytical approach of utilizing single trial data to measure within-subject trial-by-trial changes. In all succeeding models, in place of a random intercept, trial amplitude was centered at each subject's mean in order to control for the minimal between-subject differences in component amplitude. Estimates for each model are presented in Table 2.

\section{Experiment 2}

Unconditional Models: Similar to Experiment 1, the intraclass correlation for each of the temporal components was approximately equivalent, rounding to -0.08. As in Experiment 1 , a great majority of the variance in the data is accounted for by within-subject fluctuation. Trial amplitude was again centered at each subject's mean. 


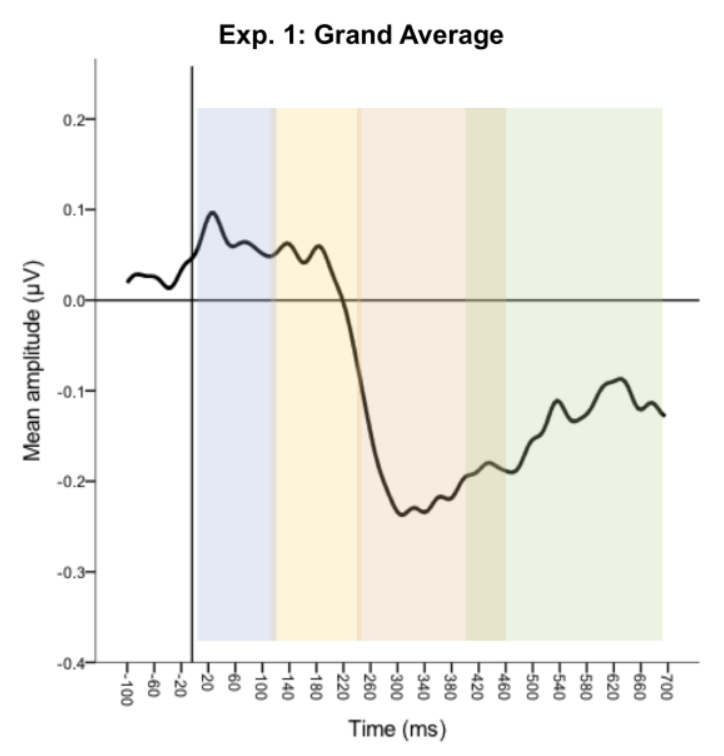

\begin{tabular}{|l|l|l|l|}
\hline HTC1 & HTC2 & HTC3 & HTC4 \\
\hline
\end{tabular}
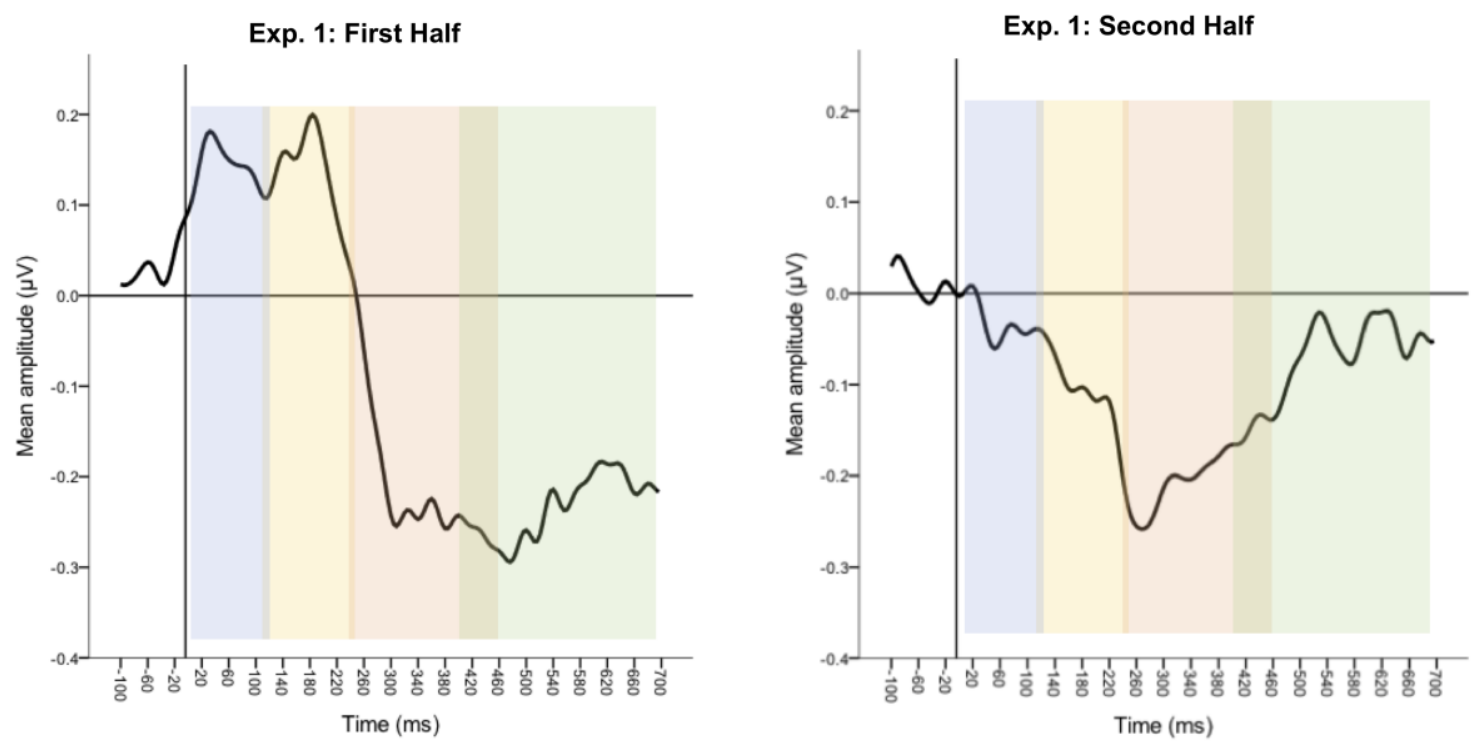

Supplemental Figure 1: Grand average ERPs for Experiment 1

Top panel: Grand average ERP responses for all trials in Experiment 1, marked with time windows for each of the temporal components of interest in shaded color bands. Bottom left: Grand averaged ERP for the first half of Experiment 1 (trials numbered 1-20). Bottom right: Grand averaged ERP for the second half of Experiment 1 (trials numbered 21-40). 

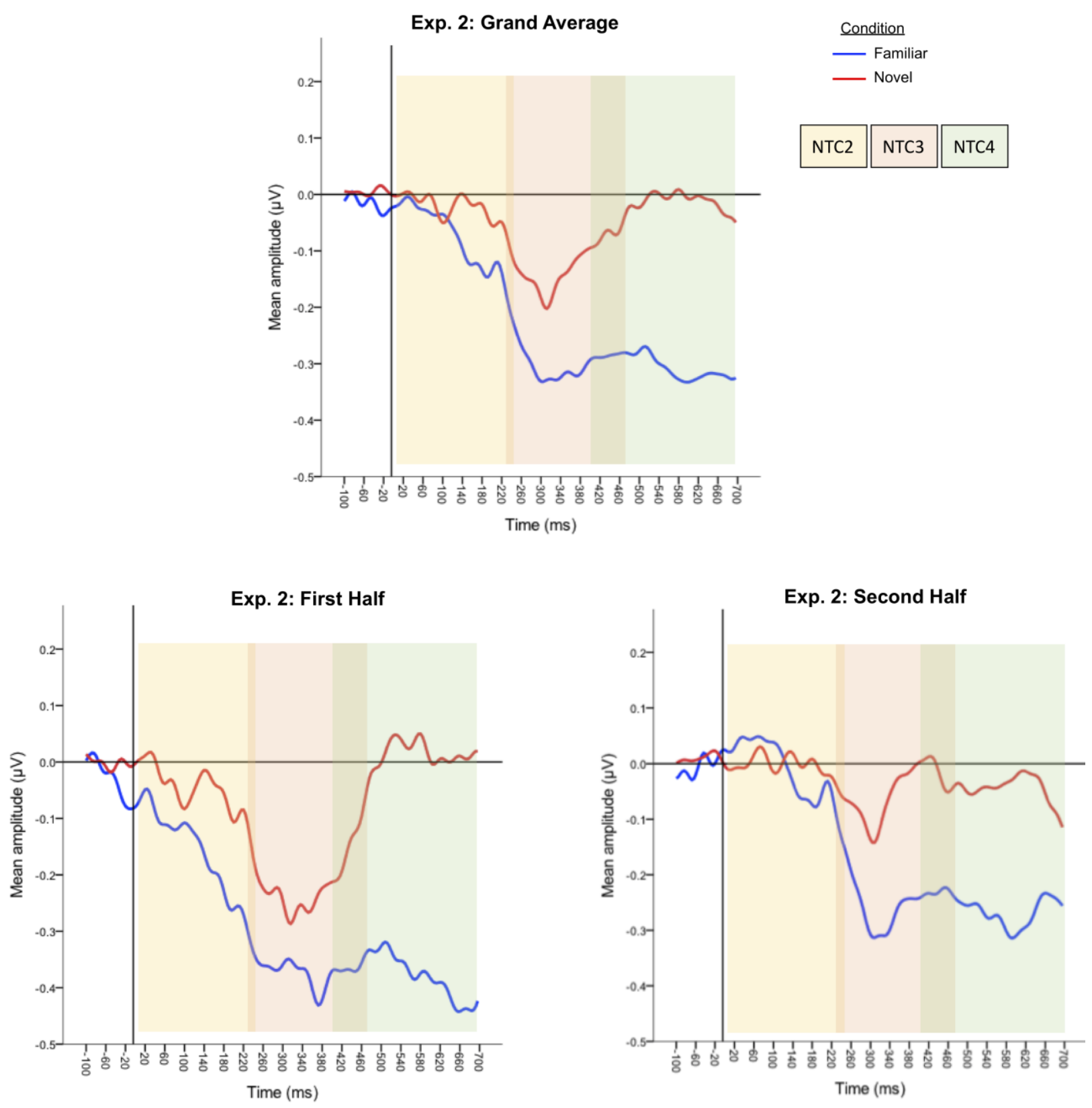

Supplemental Figure 2: Grand average ERPs for Experiment 2

Top panel: Grand average ERP responses for all trials in Experiment 2, marked with time windows for each of the temporal components of interest in shaded color bands. Bottom left: Grand averaged ERP for the first half of Experiment 2 (trials numbered 1-40). Bottom right: Grand averaged ERP for the second half of Experiment 2 (trials numbered 41-80). 